\title{
Congenital Dislocation of the Patella - Surgical Treatment Rationale and Literature Review
}

\author{
Luisella Pedrotti ${ }^{1}$, Barbara Bertani ${ }^{1}$, Federica De Rosa ${ }^{1}$, Federica Fossati ${ }^{1}$, Margherita Ghilotti ${ }^{1}$, \\ Redento Mora ${ }^{1}$, Gabriella Tuvo ${ }^{1}$, Mario Mosconi ${ }^{1}$
}

Learning Point of the Article:

Our case documents that carrying out the surgery in an older child (when it is underlined by the literature that the results are good only if the child is small) can still lead to a good result.

\section{Abstract}

Introduction: Congenital dislocation of the patella (CDP) is a rare condition and its treatment is not well defined. In CDP, patella is dislocated on the lateral aspect of the distal femur, laterally from the trochlear groove, it cannot be reduced manually and it is almost always associated with genu valgum, lateral torsion of the proximal tibia, and flexion contracture of the knee. This condition is present at birth, but the clinical findings can be subtle at birth due to the size and character of the structures being examined; early diagnosis is essential, but it may be delayed to late childhood or adulthood. If CDP is not promptly treated, the disability increases during growth, so surgical correction should be planned as soon as the diagnosis is confirmed.

Case Report: We report a case of bilateral CDP associated with bilateral proximal radioulnar joint stiffness; the patient at the age of diagnosis was 11 years old and underwent a bilateral delayed surgical procedure; the 4-year follow-up results are satisfactory. Surgical treatment rationale and literature are reviewed.

Conclusion: Surgical correction for CDP is generally recommended, but there is no agreement in literature on the ideal treatment and in some cases, with mild impairment of the knee function, observation alone has been suggested. The decision about a surgical treatment can be difficult and depends on the degree of disability of the patient.

Keywords: Congenital dislocation of the patella, bilateral congenital dislocation, patella, Z-plasty quadriceps muscle, lateral retinaculum release, lack of active knee extension.

\section{Introduction}

Congenital dislocation of the patella (CDP) is a rare congenital abnormality often familiar and bilateral; its prevalence is not known. It can be an isolate disorder or associated with other malformations of the musculoskeletal system in a complex syndrome. In the presence of CDP, genetic disorders such as arthrogryposis, lateral femoral condyle aplasia, nail patella syndrome, genitopatellar syndrome, Meier-Gorlin syndrome, Down syndrome, or others should be excluded $[1,2]$. CDP is a permanent and irreducible dislocation of the patella, resting adjacent to the lateral femoral condyle with empty trochlear groove, knee flexion contracture, or inability to achieve a full active extension; it is associated with varying degrees of genu valgum and proximal tibial torsion $[3,4]$. The most accepted etiological hypothesis is failure of the rotation of the myotome containing the quadriceps and patella which normally occurs in the first trimester of intrauterine life; if this fails to occur the extensor apparatus remains in the lateral position with permanent patellar dislocation: In this position, the extensor apparatus works as a knee flexor and external rotator $[1,5]$. This condition must be differentiated to the developmental forms of patella dislocation: It occurs toward the 8-10th weeks of embryonic development and it manifests immediately after birth [6]. In some cases, however, diagnosis may be missed early

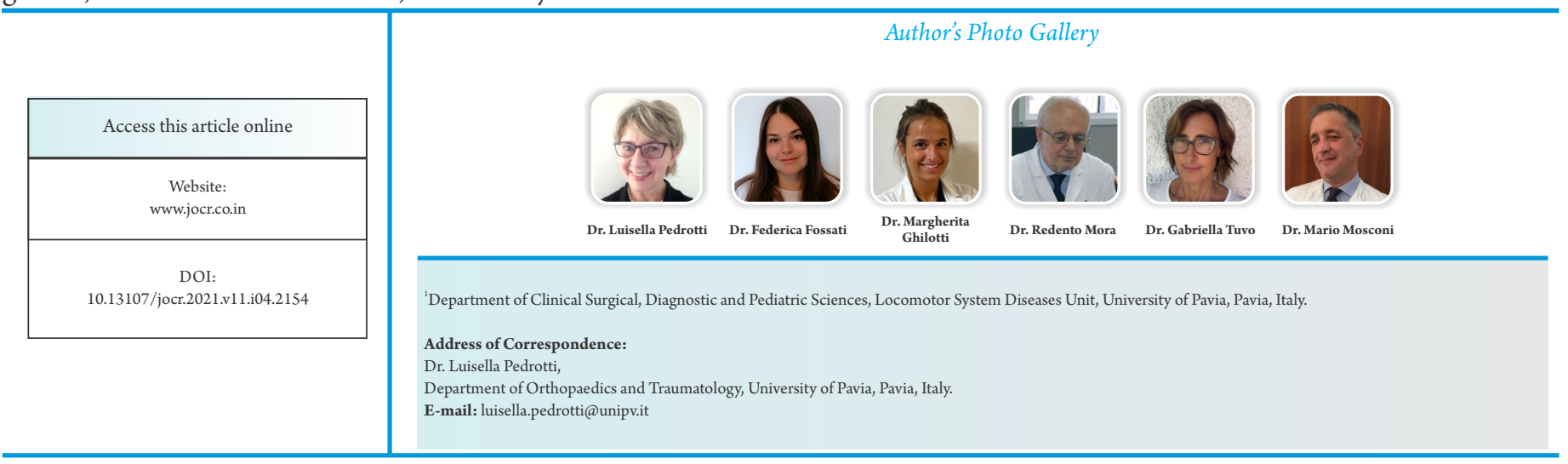

Journal of Orthopaedic Case Reports | pISSN 2250-0685 | eISSN 2321-3817 | Available on www.jocr.co.in | doi:10.13107/jocr.2021.v11.i04.2154 This is an Open Access article distributed under the terms of the Creative Commons Attribution Non-Commercial License (http://creativecommons.org/licenses/by-nc/3.0) which permits unrestricted non-commercial use, distribution, and reproduction in any medium, provided the original work is properly cited. 


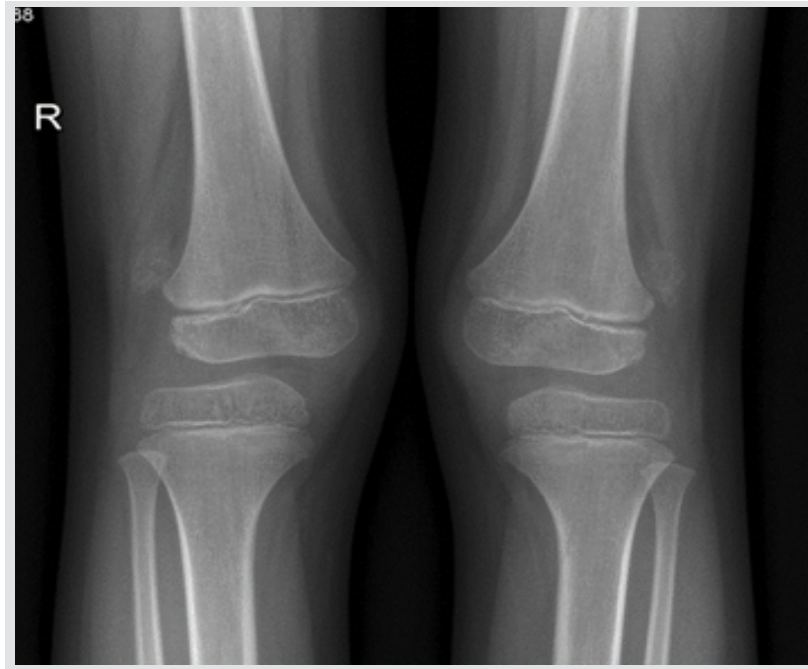

Figure 1: Pre-operative anteroposterior view of bilateral knees. X-ray before the firs operation show dislocated small patella and bilateral femorotibial rotatory malalignment.

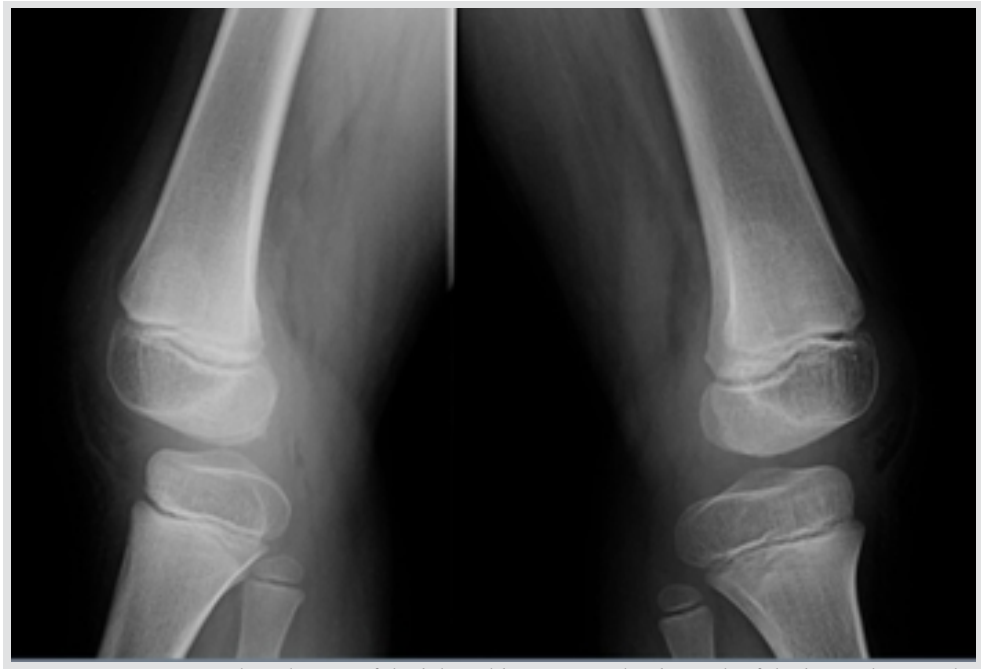

Figure 2: Pre-operative lateral views of the bilateral knees. Lateral radiograph of the knees showing the marked lateral rotation deformity of the tibia. Anterior subluxation of femur on tibia is noted. Patella is in its place, even if it is not discernible as the image overlapping femoral metaphysis.

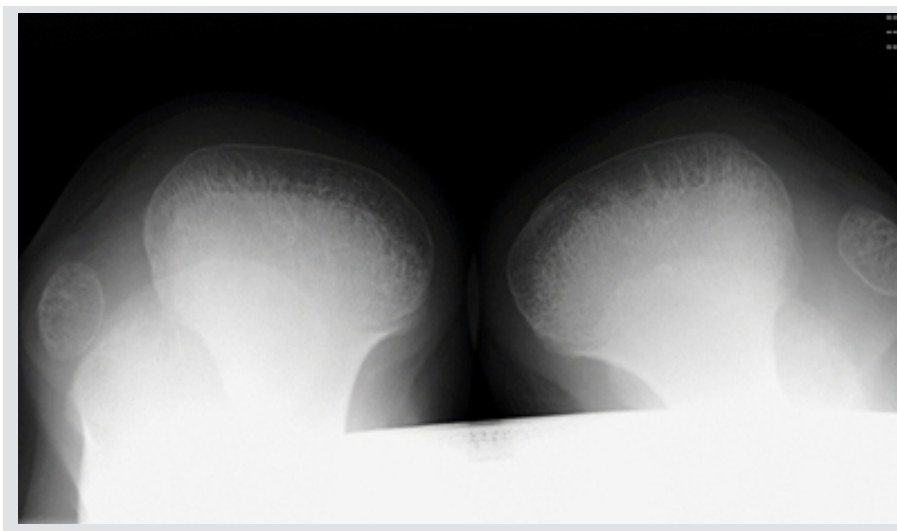

Figure 3: Pre-operative axial projection X-ray of both patellae. The patella is located at the lateral aspect of the femoral condyle, and both femoral trochleae were dysplastic.

because the dislocated patella will not be seen on plain radiographs until the patella ossifies at around 3 years of age, before that ultrasound is a better imaging study $[7,8]$; diagnosis can be delayed to late childhood, adolescence, or adulthood. If the pathology is not treated, disability increases and may lead to important degenerative changes and functional severe impairment of the knee joint. Surgical correction is generally the recommended treatment $[2,5,9]$.

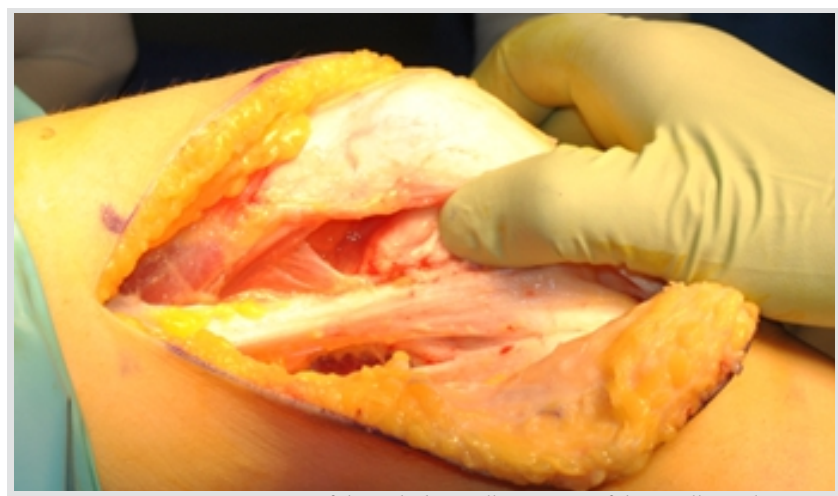

Figure 5: Intraoperative picture of the right knee. Illustrations of the patellar realignment procedure. After extensive capsular lateral release, the tight iliotibial band and the biceps femoris tendon are exposed and divided obliquely.

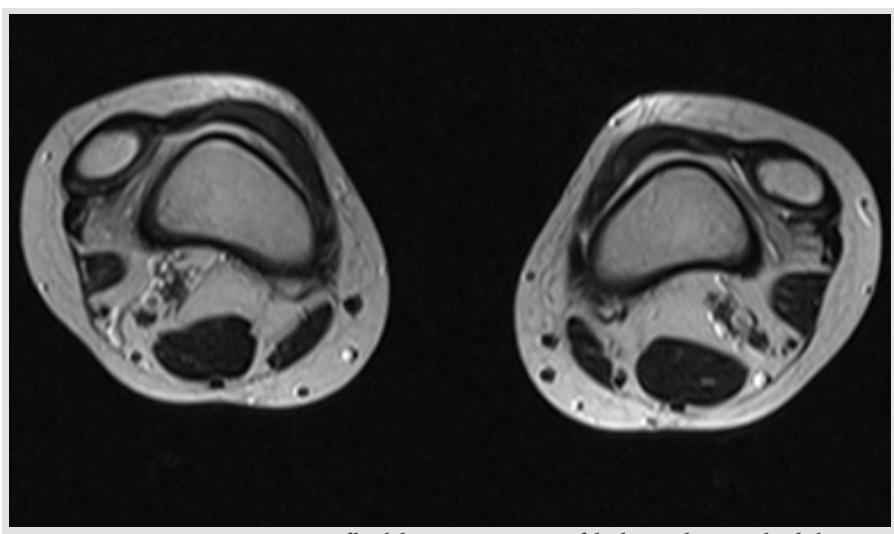

Figure 4: Pre-operative MRI image of both knees. MRI image of the knees showing the dislocation of the patellae; both quadriceps were hypotrophic, the vastus medialis enveloped over the trochlear groove, which appeared flattened and dysmorphic.

\section{Case Report}

The patient was an 11-year-old Caucasian boy without any relevant familiar history or comorbidity. He referred to our clinic complaining disability in walking and running with frequent falls (due to the lack of power in knee extensor mechanism). His parents said that despite the absence of pain,

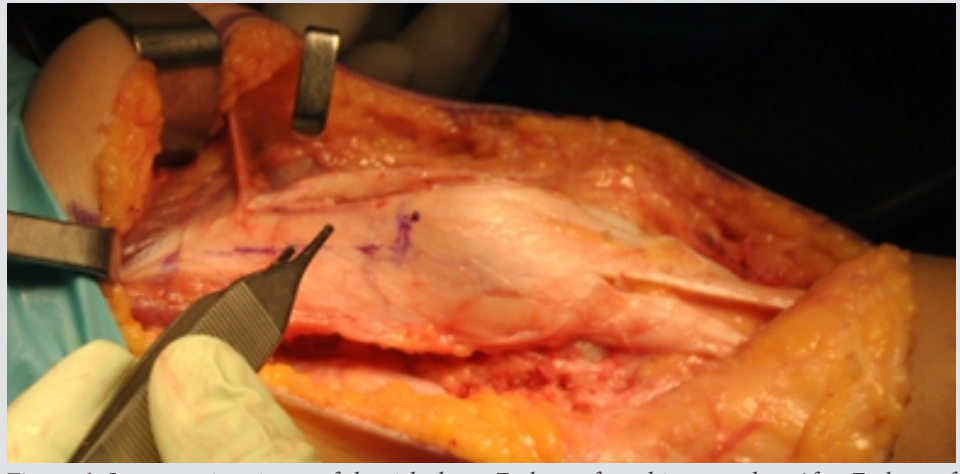

Figure 6: Intraoperative picture of the right knee. Z-plasty of quadriceps tendon. After Z-plasty of quadriceps tendon, the procedure continues with longitudinal division of the patellar tendon and suturing lateral portion medially as far as possible (Goldthwait procedure) 


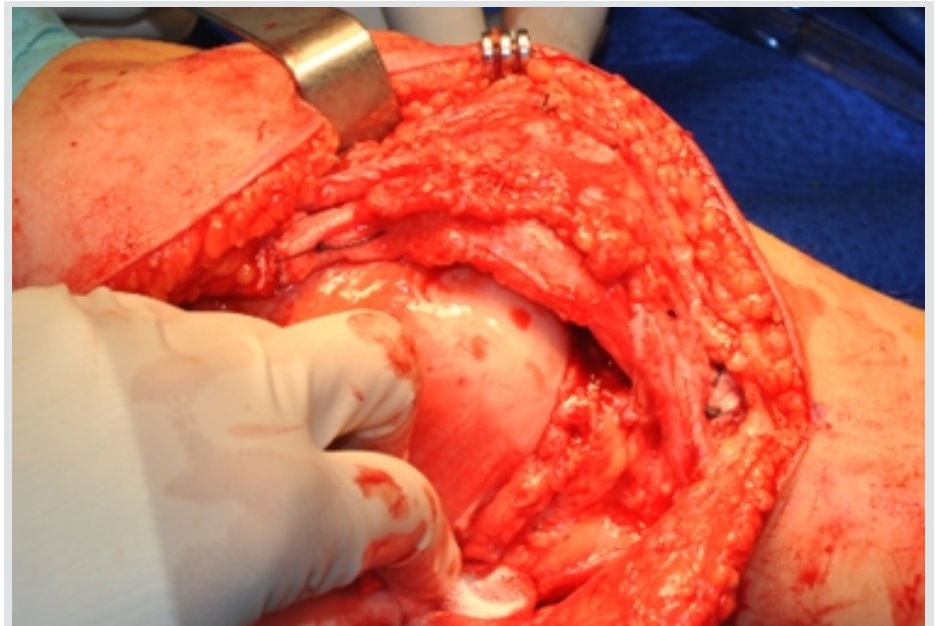

Figure 7: Intraoperative picture of the right knee. After completing the Goldthwait procedure and quadriceps lengthening, the patella is tucked under the capsule, and its medial border is sutured as far medially as possible to the capsule.

the functional limitation of both his knees was at the base of a serious psychological discomfort, conditioning his relationship with peers.

On objective examination, his lower limbs revealed mild genu valgum and tibial proximal lateral torsion. Both patellae were on the lateral aspect of the femoral condyle displacing even further during knee flexion. Femoral quadriceps function was present but this muscle was working as a flexor instead of extensor of the knee. It was observed that he was unable to actively extend both knees from the flexed position; passive range of motion (ROM) was complete and was not painful. At the upper limbs, there was a mild deficit in ROM of both elbows. There were no pathological deviation of the spine; normal clinical finding of both feet. The anterior-posterior lateral and axial knee X-rays showed bilateral, superolaterally displaced and hypoplastic patellae and reduction of the trochlear groove (Fig. 1,2,3).

The extensor mechanism was studied by magnetic resonance imaging (MRI): Both quadriceps were hypotrophic with the vastus medialis enveloped over the trochlear groove which appeared flattened and dysmorphic. The patellar tendons were bilaterally lateralized and stretched. The anterior cruciate ligament could not be identified. On both sides, MRI confirmed the lateral dislocation of the patellae (Fig. 4).

Elbows X-ray showed a slight posterior dislocation of the radial

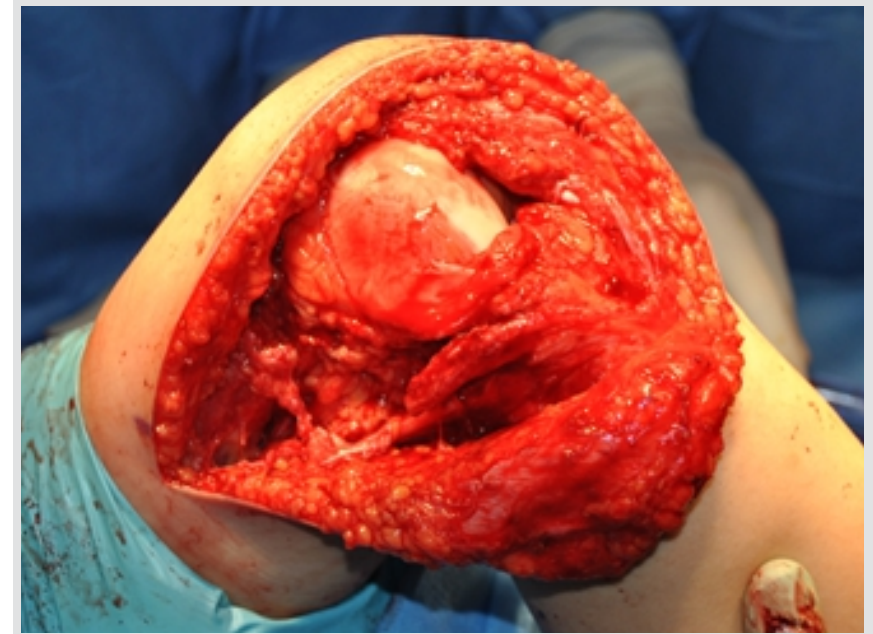

Figure 8: Intraoperative picture of the right knee. At the end of the operation, the patella appears centered in the femoral trochlea during knee flexion.

head and absence of radioulnar synostosis. The diagnosis of CDP was confirmed. Surgical correction of the deformity was then proposed to regain walking and running function, with the aim of reduce the grade of the deformity improving the patient quality of life and his psychological well-being.

\section{Surgical technique}

Surgical correction of CDP was first performed on the right knee; about 1 year later on the opposite side. Under general anesthesia, the patient in supine position, a curved skin incision was made from the lateral aspect of the distal femur to $2 \mathrm{~cm}$ below the anterior tibial tuberosity. Skin, subcutaneous tissue, and fascia lata were dissected exposing the patella retinaculum. The patella rested on the lateral aspect of the lateral femoral condyle. An extensive lateral retinaculum release was performed. The adhesions between the anterolateral aspect of the quadriceps muscle and the iliotibial band were released associated with a Z-plasty lengthening of both these structures (Fig. 5, 6).

The patellar tendon was inserted in a more lateral position: The tendon was released from its adhesion, its lateral half carefully disconnected and it was then transferred medially and secured by transosseous suture at the midline of the upper tibia. After medial retinaculum and capsular release, the patella could be

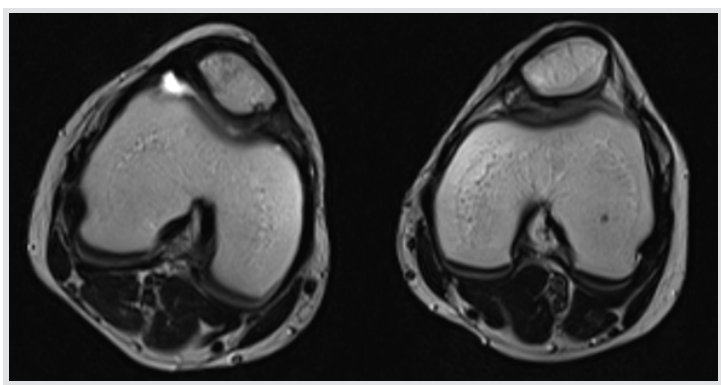

Figure 9: MRI at 4 years of follow-up. MRI images showing the reduced patella in the groove of the femoral condyle.
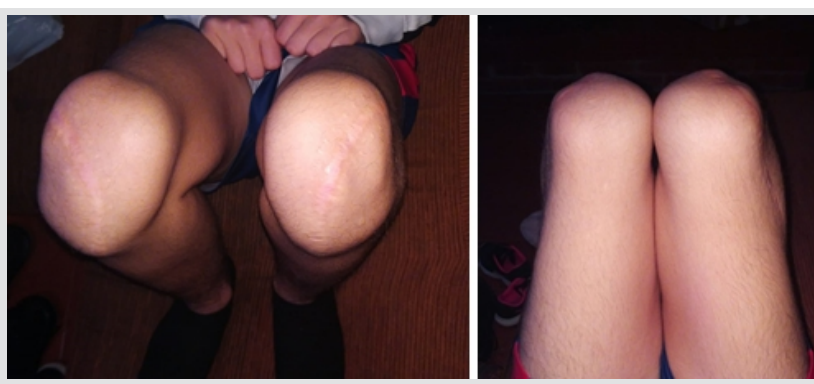

Figure 10: Clinical images of post-operative range of motion.
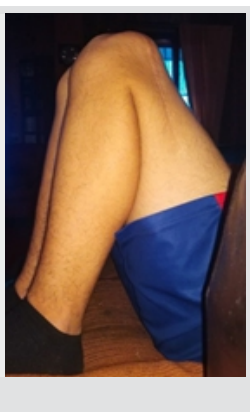
reduced in place and fixed under the medial capsular surface (Fig. 7).

The patella stability in flexion and extension was checked carefully (Fig. 8).

In the right knee, patella's stability was not acceptable, so a plication of the vastus medialis tendon was added to the procedure to increase the stability. The skin was closed and a drainage maintained in place for 2 days.

\section{Post-operative follow-up}

A well-padded long leg plaster cast was applied with $20^{\circ}$ of knee flexion for 6 weeks. It followed an uneventful post-operative course. After cast removal, a patellar centralizing brace was applied and active and passive mobilization and quadriceps strengthening exercises FKT protocol were started. At 7 months' post-operative follow-up consultation, both patellae were centered without any episode of acute/subacute dislocation and showed complete extension and $120^{\circ}$ of flexion on both knees (Fig. 9).

Four years of follow-up results may be considered satisfactory. During the last clinical evaluation, active knee extension was restored despite a residual Grade IV strength deficit in the left leg extension (Fig. 10).

\section{Discussion}

CDP is a permanent and irreducible condition often present at birth and it must be distinguished from recurrent habitual or permanent but reducible dislocation $[1,5]$. In the developmental form, the patella dislocation is not permanent and is reducible: The patella drifts laterally during knee extension and this condition usually becomes clinically evident when the child starts to walk [9]. Some authors suggest that all of these conditions represent different degrees of the same pathology since they found the coexistence of congenital and recurrent patellar dislocation in the same patient or in the same family $[2,10]$. Cadaver studies carried out by Ghanem et al. do not support this theory and designed CDP as a different pathogenic entity [11]. This condition occurs toward the $8-10$ th weeks of embryonic development and it can manifest immediately after birth; Stanisavljevic et al. suggested as a possible pathogenesis a failure in the internal rotation of the myotome containing the quadriceps muscle with the patella resting in the anterolateral aspect of the femur [1].

Some patients, especially when CDP is part of a syndromic disease, are easily diagnosed at birth. Isolated patella dislocation must be suspected in newborn with knee flexion contracture; however, objective clinical examination of infant's knee is often difficult and the palpation of even a normal patella can be uncertain. Furthermore in infants, the patella begins to ossify at almost 3 years of age [6]. Hence, when clinical suspected, CDP can be diagnosed only by sonography. In older children and adolescents conventional X-ray in anteroposterior, lateral and axial view can show the position, size and shape of the patella, the hypoplastic femoral groove and lateral condyle, and the severity of the lateral joint space narrowing. MRI is essential for identifying and characterizing lesions associated with permanent dislocated patella (soft-tissues structures of the extensor mechanism, chondral lesions) but sedation in younger patients may be required $[8,12]$.

The disease produces varying degrees of knee disability that may increase with child's growth. In some cases, walking is very difficult, sometimes impossible; closed reduction is universally unsuccessful and operative management is considered the mainstay of treatment for most of the authors. Several different surgical techniques are described but all follow the same principles: Extensive lateral release to allow centralization of the patella; V-Y quadricepsplasty or Z-lengthening of the extensor mechanism and imbrications of the redundant medial capsule to stabilize the patella. Many authors recommend surgery during childhood considering unpredictable the outcomes in older children $[4,5,13,14,15]$.

McCall and Lessenberry reported a case of a 19-month-old girl with by bilateral CDP who underwent Stanisavljevic procedure with excellent results [4].

Gao monitored 12 patients (average age of 5 years) who underwent extensive external release and realignment of the extensor apparatus for $\mathrm{CDP}$, with about $85 \%$ of positive result at follow-up ranging from 2 to 15 years (average 5 years).

Gordon reported 10 patients (average age at presentation 7 years and 9 months) who underwent an extensive procedure including lateral release, advancement of the vastus medialis, oblique, and medial transfer of the patellar tendon. At last follow-up, all patients reported relief of pain and functional improvement [15]. Langenskiöld and Ritsilä described a series of 12 patients ( 18 knees) in whom the diagnosis of CDP had been delayed and the surgical procedure had consequently been carried out from 3 to 14 years of age; based on the clinical outcomes, the author concluded that early surgical treatment leads to better results but the intervention can also be justified in older patient [14].

Most patients in previous review had a flexion knee contracture with difficult walking. It is reported that when the ability to extend the knee is preserved, CDP does not lead to functional failure and it is likely that the pathology comes at the attention of the physician during the adulthood $[3,11,16,17,18]$. 
Tokgöz described a case of a 16-year-old patient with neglected bilateral CDP who presented with complaints of gait disturbance, frequent falls, and muscle weakness in the lower limbs without ROM limitation. Due to the absence of pain and limitation in joint movement, no surgical indication was given which the patient would have refused anyway [16].

Illyas reported the case of bilateral CDP with synostosis of proximal tibiofibula, proximal radioulnar joint in a 30-year-old man which underwent extensive lateral retinacular release when he was 13 years old. The patient complained of pain and recurrent dislocation of the left patella but he did not accept a further surgical treatment as his pain was not affecting his daily life [17].

Bistolfi et al. described the case of a 51-year-old patient suffering from bilateral CDP who complained of knee pain after a meniscal injury; no treatment of the patella dislocation had been proposed. The authors reported other cases of CDP diagnosed and treated in adulthood with varying results [3].

Yamanaka et al. described the case of a patient suffering from CDP and undergoing knee arthroplasty at the age of 70 with a reduction in patella dislocation on one side only [18]. Knee flexum rather than extensor mechanism insufficiency seems the discriminating factor in the choice of treatment and soft-tissue surgery of realigning the extensor apparatus in the first-line treatment in symptomatic children and adolescent [20].

In our case, functional disorder was associated with impaired quadriceps function affecting long distance walking and sports; there was no pain or contracture in knee flexion, for this reason, an invasive procedure was barely acceptable for the family; on the other hand, disability had caused a progressive removal of the child from peers with a personality disorder that required psychologist help. Treatment of DCP is neither urgent nor mandatory. The legal guidelines are that the parent must decide in the interest if the child; the physician should attempt to include the child in the decision process even if his judgments depends on the age. For children younger than 12 or 13 years of age, there is probably a little decision ability, but it is appropriate talking directly to the child about the potential treatments [20]. The therapeutic strategy must be shared after the risks and benefits have been carefully illustrated as, despite CPD is not a serious disease, patellar realignment can only be obtained surgically with an invasive and complex treatment. We believe that the surgical procedure for CDP should be proposed in patients with impaired quality of life and, based on our results, it can also be performed with good outcomes in older children.

\section{Conclusion}

$\mathrm{CDP}$ is a permanent and irreducible condition often present at birth and it must be distinguished from recurrent habitual or permanent but reducible dislocation. The disease produces varying degrees of knee disability that may increase with the Clinical Message

Based on our experience, we believe that surgical treatment, in the presence of functional or psychological disorders, can give good results even in the older child.

Given the rarity of the disease, most scientific publications consider the description of a single patient, as we did. The discussion was whether to face the surgical correction immediately (by carrying out a very invasive intervention early, at the year of life, as recommended by many publications) or whether to completely neglect the problem (as documented by the other case described in the text, in which the adult patient was diagnosed only on the occasion of a trauma and the patient underwent only meniscectomy, continuing his activity regularly). Another scientific publication takes into account the case of a patient in whom the reduction was performed only on the occasion of the knee prosthetic replacement, so the surgery can be delayed or never be performed. What to do? Our case documents that carrying out the surgery in an older child (when it is underlined by the literature that the results are good only if the child is small) can still lead to a good result. We presented it as a case report, that is why we make these conclusions.

\section{References}

1. Stanisavljevic S, Zemenick G, Miller D. Congenital, irreducible, permanent lateral dislocation of the patella. Clin Orthop 1976;116:190-9.

2. Jones RD, Fisher RL, Curtis BH. Congenital dislocation of the patella. Clin Orthop Relat Res 1976;119:177-83.

3. Bistolfi A, Massazza G, Backstein D, Ventura S, Cerlon R, Crova M. Adult congenital permanent bilateral dislocation of the patella with full knee function: Case report and literature review. Case Rep Med 2012;2012:182795.
4. McCall RE, Lessenberry HB. Bilateral congenital dislocation of the patella.J Pediatr Orthop 1987;7:100-2.

5. Green JP, Waugh W, Wood H. Congenital lateral dislocation of the patella.J Bone Joint Surg Br 1968;50:285-9.

6. Walmsley R. The development of the patella. J Anat 1939; 74:360-9.

7. Wada A, Fujii T, Takamura K, Yanagida H, Surijamorn P. Congenital dislocation of the patella. J Child Orthop 2008;2:119-23. 
8. Walker J, Rang M, Daneman A. Ultrasonography of the unossified patella in young children. J Pediatr Orthop 1991;11:100-2.

9. Thabit G, Micheli LJ. Patellofemoral pain in the pediatric patient. Orthop Clin North Am 1992;23:567-85.

10. Johnson EW Jr., Milicic M. Congenital dislocation of the patella.J Anat 1939;74:360-9.

11. Ghanem I, Wattincourt L, Seringe R. Congenital dislocation of the patella. Part I: Pathologic anatomy. J Pediatr Orthop 2000;20:812-6.

12. Koplewitz BZ, Babyn PS, Cole WG. Congenital dislocation of the patella. AJRAm J Roetgenol 2005; 184:1640-6.

13. Gao GX, Lee EH, Bose K. Surgical management of congenital and habitual dislocation of the patella.J Pediatr Orthop 1990;10:255-60.

14. Langenskiold A, Ritsila V. Congenital dislocation of the patella and its operative treatment. J Pediatr Orthop $1992 ; 12: 315-23$.

15. Gordon JE, Schoenecker PL.Surgical treatment of congenital dislocation of the patella. J Pediatr Orthop
1999; 19:260-4.

16. Tokgöz MA, Çavuşoğlu AT, Ayanoğlu T, Elma T, Vural A. Neglected bilateral congenital dislocation of the patella. Eklem Hastalik Cerrahisi 2017;28:128-31.

17. İlyas G, Eren TK, Kaptan AY, Ulucaköy C, Kanatlı U. Bilateral congenital dislocation of the patella associated with synostosis of proximal tibiofibular and proximal radioulnar joints: A case report. Eklem Hastalik Cerrahisi. 2018;29:123-7.

18. Yamanaka H, Kawamoto T, Tamai H, Suzuki M, Kobayashi T, Eguchi Y, et al. Total knee arthroplasty in a patient with bilateral congenital dislocation of the patella treated with a different method in each knee. Case Rep Orthop 2015;2015:890315.

19. Wang CH, Shu L, Ma LF, Zhou JW, Ji G, Wang F, et al. Medial and lateral retinaculum plasty for congenital patellar dislocation due to small patella syndrome. Orthopedics 2013;36:1418-23.

20. Lindseth RE. Ethical issues in pediatric orthopaedics. Clin Orthop Rel Res 2000;378:61-5.
Conflict of Interest: Nil

Source of Support: Nil
Consent: The authors confirm that informed consent was obtained from the patient for publication of this case report

\section{How to Cite this Article}

Pedrotti L, Bertani B, De Rosa F, Fossati F, Ghilotti M, Mora R, Tuvo G, Mosconi M. Congenital Dislocation of the Patella - Surgical Treatment Rationale and Literature Review. Journal of Orthopaedic Case Reports 2021 April;11(4):59-64. 